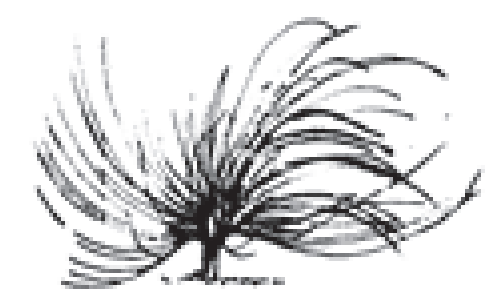

\title{
Prácticas Académicas Inclusivas en el Aula Universitaria: Un Aprendizaje Intercambiable
}

\author{
Karol Viviana Cubero Vásquez ${ }^{1}$ \\ Universidad Nacional \\ Sede Regional Chorotega \\ Guanacaste, Costa Rica \\ kacubero@gmail.com \\ Lucia Svetlana Villanueva Monge ${ }^{2}$ \\ Universidad Nacional \\ Sede Regional Chorotega \\ Guanacaste, Costa Rica \\ mongeluc@gmail.com
}

Trabajar en equipo no es una virtud, es una elección consciente y voluntaria que surge construyendo lazos de confianza basados en la vulnerabilidad humana que muestran los integrantes del equipo, ante sus errores, temores y dificultades.

Patrick Lencioni

\begin{abstract}
Resumen
En las aulas universitarias se hace necesario llevar a cabo prácticas educativas que permitan a todos los estudiantes participar activa y equitativamente del proceso educativo. Se necesita un cambio en la metodología de enseñanza- aprendizaje tradicional para hacer de este proceso uno más cooperativo, inclusivo, dinámico y justo, el cual
\end{abstract}

Recibido: 12 de marzo de 2014-Aprobado: 25 de octubre de 2014

1 Magister en Educación con mención en Inglés, se desempeña como profesora en la enseñanza del Inglés en la Universidad Nacional. Posee una maestría en Educación con énfasis en el Aprendizaje del Inglés de la Universidad Nacional.

2 Magister en Educación con mención en Inglés, se desempeña como profesora en la enseñanza del Inglés y en turismo sostenible en la Universidad Nacional. Posee una maestría en Educación con énfasis en el Aprendizaje del Inglés en la Universidad Nacional. 
se base en un aprendizaje solidario que busque el trabajo en equipo y ayuda entre pares con tutorías realizadas por los mismos estudiantes para sus propios compañeros. Esta práctica beneficia no solo al estudiante con dificultades que aprende con el apoyo directo de uno de sus compañeros, sino que trae ventajas también al estudiante que enseña, pues con esta acción, está reafirmando conocimiento y entonces, cuando enseña, aprende. Se promueve así valores como la solidaridad, la cooperación, la responsabilidad, la armonía y la amistad entre todos los actores del proceso. En este documento se establece el aprendizaje cooperativo a través de la práctica de actividades que conlleven el trabajo en equipo y las tutorías entre estudiantes o pares, con el propósito de lograr un aprendizaje significativo, efectivo e interactivo que contribuya a una formación más integral del aprendiz.

Palabras clave: prácticas inclusivas, trabajo cooperativo, tutorías, actividades

\begin{abstract}
In our university classrooms, there is an imperative need for implementing new educational practices that allow all students to participate actively and equally in the learning process. A change is required in the teaching-learning methodology to assure a more cooperative, inclusive, dynamic, and fair learning process based on by team work and peer support with tutoring given by students to their classmates. This practice not only benefits the student facing some difficulties, who learns with the support of one of his/her classmate but also brings advantages to the student that teaches because with this, knowledge is reinforced, and thus when he or she teaches, he or she learns. When this practice is promoted, values such as solidarity, cooperation, responsibility, harmony, and friendship are shared among all the participants involved in the process. This article supports a cooperative learning process enriched with activities that value team work and tutoring among students, seeking a significant, effective, and interactive learning process that can lead to a more integral education of the learner.
\end{abstract}


Keywords: inclusive practices, cooperative work, tutoring, activities

\section{Introducción}

U

na de las dificultades que experimentamos como docentes en el aula universitaria al momento de realizar una actividad didáctica, es la renuencia de muchos de nuestros estudiantes a trabajar en grupo, con compañeros con los que nunca antes habían trabajado, o peor aún, esta renuencia es aún mayor cuando les corresponde trabajar en equipo con compañeros que han demostrando más dificultades para aprender ciertos contenidos durante el curso.

Por ello es necesario hacer conciencia, tanto en los educadores como en los educandos, de la importancia de llevar a cabo prácticas educativas que permitan incluir a todos los estudiantes en el proceso educativo. Se necesita un cambio en la metodología de enseñanzaaprendizaje tradicional para hacer de este proceso uno más cooperativo, inclusivo, dinámico y justo, el cual se base en un aprendizaje solidario y de calidad, que busque el trabajo en equipo y ayuda entre pares, con tutorías realizadas por los mismos estudiantes para sus propios compañeros. Al decir ayuda entre pares, se habla de un estudiante enseñando a otro quien puede presentar más dificultades para aprender un conocimiento específico. Esta práctica beneficia, no solo al estudiante con dificultades, sino que favorece también al estudiante que enseña, pues con esta acción, está reafirmando conocimiento y entonces, cuando enseña, aprende. Apelamos aquí, no solo el sentimiento de solidaridad, cooperación y de responsabilidad por parte del estudiantado, sino también, se pretende hacer comprender que con la práctica del trabajo entre pares, tutorías estudiantiles y trabajo en equipo, se da un beneficio intercambiable: "usted me da, yo le doy." Es un aprendizaje equitativo y justo.

Es así que en este documento, se realiza una revisión de la experiencia costarricense en la implementación de la educación inclusiva hasta nuestros días; así como su normativa y práctica. Asimismo, se analiza lo que define la educación inclusiva según importantes investigadores, estudiosos de la materia, estableciendo sus características e implicaciones en la educación e incluyendo, por supuesto, la metodología de aprendizaje cooperativo, pilar fundamental de la educación inclusiva. 
Aunado a lo anterior y como ejercicio del aprendizaje cooperativo, se plantea en este artículo actividades didácticas que conllevan el trabajo en equipo y las tutorías entre estudiantes o pares, partiendo de una experiencia de inclusión vivida con un grupo de estudiantes universitarios en los cursos de inglés como lengua extranjera y en los cuales hemos sido las profesoras a cargo. Todo esto con el propósito de lograr en nuestros educandos un aprendizaje significativo y efectivo que, al mismo tiempo, promueva satisfacción por logros académicos alcanzados y mejore la percepción propia del estudiante y de sus compañeros; para así contribuir a su madurez emocional con motivación, amistad, armonía e interacción en el aula universitaria.

\section{Sustentos teóricos}

\section{Normativa: Derechos de inclusión}

Según la UNESCO (2009), la educación inclusiva es un proceso que pretende acoger la diversidad y las necesidades de todos los y las estudiantes mediando la cultura y la comunidad en participación activa, con el fin de disminuir la exclusión en y desde la educación. La educación inclusiva aspira a lograr una educación de calidad para todos, asegurando acceso, permanencia, participación y logros en los objetivos de aprendizaje (p. 8).

Esto pone de manifiesto la necesidad de direccionar esfuerzos que garanticen una educación equitativa que respete las diferencias e incluya oportunidades de participación pensadas hacia un aprendizaje en interacción con los demás. La educación inclusiva es un derecho humano por lo que no se debe aislar a nadie como consecuencia de sus diferencias (discapacidad, dificultad de aprendizaje, género o pertenencia a una minoría).

En Costa Rica la Ley 7600 (1996), en relación con el acceso a la educación artículo 14, indica que el Estado garantizará el acceso oportuno a la educación (desde la estimulación temprana hasta la educación superior), a todas las personas, independientemete de su condición o discapacidad. En el artículo 17 sobre adaptaciones y servicios de apoyo indica que los centros educativos efectuarán las adaptaciones necesarias y proporcionarán los servicios de apoyo requeridos para que el derecho de las personas a la educación sea efectivo. Se enmarcan en estas disposiciones, adaptaciones 
curriculares, recursos humanos especializados, evaluaciones, metodología, recursos didácticos y planta física (p. 6).

Han transcurrido 18 años de la creación de esta Ley y su reglamento (promulgado en 1997), dando legalidad y obligatoriedad en brindar atención a las necesidades especiales en las instituciones públicas y privadas del país. En este sentido, esta Ley ha llevado a la práctica educativa la aplicación de las adecuaciones curriculares en nuestras aulas con todas sus implicaciones en recursos humanos y didácticos. De acuerdo con el IV informe del Estado de la Educación, Desarrollo y Desafíos de las Adecuaciones Curriculares en el Sistema Educativo Costarricense, documento tutelado por el Consejo Nacional de Rectores de Costa Rica (CONARE), se manifiesta el conocimiento de la obligatoriedad de aplicar las adecuaciones curriculares y sus diferentes tipos, estableciendo muy claramente que las adecuaciones curriculares son un derecho de los educandos. Según Meléndez et al. (2012), desde el año 1999 al año 2012, se han triplicado las adecuaciones curriculares no significativas en todo el territorio nacional y los números van en ascenso. Sin embargo, aún se plantean muchas interrogantes por parte de los educadores en cuanto a la manera de cómo se deben aplicar; es decir, su operación y práctica, lo que a su vez es percibido por muchos, como un trabajo extra dentro de sus tareas como educadores y en el cual los recursos son aún limitados (pp. 05- 06).

Entonces, en Costa Rica, las adecuaciones curriculares representan los esfuerzos realizados en este campo. Según Meléndez et al. (2012), nuestro país se encuentra en un proceso de transición de un enfoque de integración con adecuaciones curriculares como eje principal de apoyos educativos a uno realmente inclusivo en la educación, con una educación más equitativa y de calidad y utilizando las adecuaciones curriculares como una herramienta más a utilizar ante las diferencias personales y culturales. Es así como emerge una nueva propuesta redactada en la más reciente ley 8661, de la Convención de los Derechos de las Personas con Discapacidad y la Política Nacional en Discapacidad 2011-2021 (PONADIS), en la que se empieza a gestar un cambio, considerando a las adecuaciones curriculares como una alternativa de la educación inclusiva pero con un carácter más social, considerando la diversidad como parte inherente de la naturaleza humana (p. 9).

Partiendo de este nuevo principio, es que las universidades públicas costarricenses como la Universidad Estatal a Distancia, la 
Universidad Nacional de Costa Rica, la Universidad de Costa Rica y otras instituciones como el Consejo Nacional de Rectores, el Colegio de Licenciados y Profesores y el Ministerio de Educación Pública han gestado esfuerzos conjuntos, involucrándose más para lograr el desarrollo y la implementación de mejores prácticas a favor de la educación inclusiva. Por ello se creó, en el Ministerio de Educación Pública, la Comisión Nacional de Educación Inclusiva en la Dirección de Desarrollo Curricular de este Ministerio. Estas iniciativas son aún incipientes, pero están en proceso de crecimiento; recordando que la educación inclusiva, por su naturaleza comprensiva, es más compleja y debido a esto, requiere de una mayor investigación, innovación y de un cambio estructural desde la misma concepción tradicional de la educación.

\section{La educación inclusiva}

Como se mencionó con anterioridad, en nuestro país es evidente el creciente interés y necesidad de concretar acciones pertinentes en relación con la educación inclusiva. Este interés se sustenta en varios hechos ya de por sí tangibles en diferentes ámbitos de la vida social, político, económico y escolar pero aun la educación inclusiva es considerada como un enfoque para servir a sectores con discapacidades dentro de los marcos de educación general.

Concordamos con Naradowski (2008), al plantear que "la inclusión educativa requiere esfuerzos de distintos sectores de la sociedad para brindar una educación sensible a las necesidades específicas de sectores, compensando las desigualdades, facilitando el acceso, la permanencia y el progreso a aquellos que más lo necesiten..." (Hacia una definición de inclusión educativa, parra, 2).

Una concepción sinónima la mantiene Stainback (2001), al describir la educación inclusiva como el proceso por el cual se ofrece a todos los y las estudiantes, sin distinción, la oportunidad de aprender de, y con sus compañeros, en un entorno en el que las diferencias son reconocidas y celebradas (Diversity paradigm, parra, 3 ).

Una aula inclusiva no acoge sólo a aquellos cuyas características y necesidades se adaptan a las características del aula y a los recursos disponibles, sino que es inclusiva, precisamente, porque acoge a todos los y las alumnos(as), tengan o no alguna discapacidad, motivando a que participen en las mismas actividades de enseñanza y aprendizaje. 
Para Echeita (2005), la atención a la diversidad remite en primer lugar, al objetivo de perseguir una individualización de la enseñanza en la que exista una preocupación por conocer las características personales de cada uno de los alumnos y las alumnas y se desee llevar a cabo una enseñanza que adapte o modifique los objetivos, los contenidos y la didáctica para alcanzar el mayor grado posible de ajuste y de individualización de la actividad educativa (La realidad multidimensional del concepto de atención a la diversidad, p. 2).

Hoy, nuestra realidad demanda de esta visión, amplia y justa. La educación inclusiva concierne a todos los y las estudiantes, persigue un proceso de enseñanza - aprendizaje significativo, valioso, equitativo y pertinente para todos y todas. Esto constituye un reto para la Universidad ya que cuestiona entre muchos aspectos, las prácticas académicas, el trabajo en las aulas, el currículo y aspectos organizativos de la institución. En general, exige un cambio cultural importante puesto que es común pensar que las dificultades o diferencias educativas responden a déficits de los alumnos denominándolos "alumnos con necesidades educativas especiales", o bien, se segregan a ciertos estudiantes en función de su condición económica, de etnia, lengua o género, gustos entre otros.

Nuestra realidad educativa superior, lamentablemente, no se puede denominar inclusiva en un $100 \%$. Se nos confiere la tarea de abordar esta temática con una mirada realista e integradora y de una vez por todas, sin exclusiones. En este ámbito, muchos profesores hemos sido testigos de cómo los estudiantes excluyen al educando que más ha demostrado dificultad en un área específica de estudio. Ellos mismos expresan que no les "conviene" por llamarlo de alguna manera, realizar una tarea específica asignada con el o la compañera que más le cuesta porque dicen no van "aprender nada y todo les tocará hacerlo a ellos solos", (palabras repetidas y expresadas por los mismos estudiantes). Entonces, el profesor se ve en esta dificultad, surge el reto de motivar a los estudiantes a desarrollar actividades con metodologías cooperativas ya que lamentablemente se evidencia una negatividad de los y las estudiantes de trabajar con compañeros o compañeras que presentan dificultades en un aprendizaje específico. Esto es una realidad que solo se supera con prácticas inclusivas estructuradas, planeadas y guiadas por el o la profesora a cargo.

Por ello, se destaca la urgencia de propiciar un ambiente de aula ameno y evidentemente inclusivo sin que esto signifique una carga 
extra, sino que exista un genuino interés de todos los integrantes de la comunidad universitaria en ofertar una educación superior inclusiva.

Esta consideración puede claramente fortalecerse desde las prácticas académicas que acontecen en un salón de clase universitario al considerar los siguientes aspectos:

- $\quad$ pasión por la enseñanza

- $\quad$ empatía hacia los demás

- crear entornos ricos en estímulos

- realimentación positiva

- crear oportunidades para todos

- concientizar sobre

- diversificación de las actividades académicas

- $\quad$ promoción del aprendizaje cooperativo

La atención a la diversidad del educando en la universidad nos plantea un asunto complejo, por lo tanto, requiere de prácticas honestas, pensadas para incluir a todos y todas. El trabajo en el aula debe, conscientemente, mediar esfuerzos que verifiquen el cumplimiento de la normativa inclusiva ofreciendo espacios a los estudiantes para aprender a aprender en comunidad, juntos, promoviendo el agrado y aceptación de diferencias, características y particularidades propias.

\section{Aprendizaje cooperativo}

El ser humano es un ser social, el desarrollo de su integridad personal y profesional depende de la interacción y convivencia con las demás personas. Nuestra interdependencia es tal que solo logramos ser felices cuando compartimos nuestras experiencias individuales con otros. De ese mismo modo sucede en el aprendizaje, en la dimensión individual se desarrolla gracias a la cooperación con otros, bajo una meta en común que se establece previamente.

El aprendizaje cooperativo es una plataforma que muy acertadamente vincula el rendimiento personal y el grupal llevado a un nivel intercambiable en el que varios elementos convergen al mismo tiempo como la cooperación, el respeto y la diversidad.

El aprendizaje cooperativo planteado por Johnson y Johnson (1999), promueve el trabajo en grupos en los que las y los alumnos 
trabajan juntos para maximizar su propio aprendizaje y el de los demás, motivando la participación equitativa para que todos los miembros del grupo tengan las mismas oportunidades de participar bajo un marco de interacción simultánea (p. 17).

Todos estos autores conciben al aprendizaje cooperativo como herramienta que más allá de organizar la clase en grupos, de gestionar tareas académicas y de motivar la socialización. La interacción e intercambio funcionan como plataforma para obtener diversos principios cognitivos, pedagógicos e inclusivos.

Estas prácticas deben de caracterizarse por una constante comunicación que derive en la activa construcción de conocimiento desde diferentes perspectivas y desde los diferentes actores inmersos en las prácticas académicas propuestas por el facilitador de prácticas académicas cooperativas -inclusivas. Para que esas prácticas alcancen los propósitos mencionados se deben asegurar los siguientes elementos a lo interno del equipo.

\section{Figura 1. Elementos cooperativos}

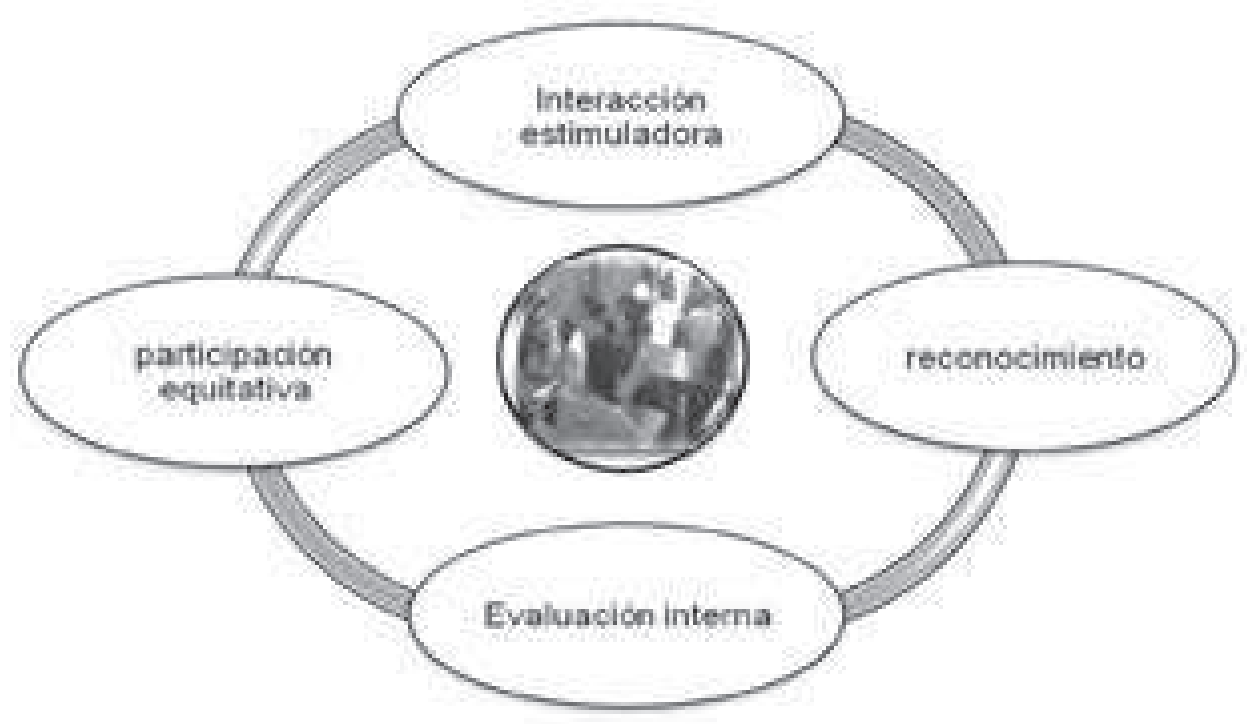

Fuente: Adaptado de Kagan 2001, Six important differences, parraf.10 
Estos apuntes tienen la clara finalidad de mediar procesos de enseñanza- aprendizaje en la que todos los miembros de un equipo sean protagonistas de un aprendizaje significativo e integrador en la que se fusionen estos elementos. Propiciando un trabajo cooperativo equitativo desde una interacción que estimule a los integrantes a realizar la tarea académica propuesta, por ende los objetivos, no solo académicos, sino también sociales. Para que esta interacción sea fructífera, el monitoreo constante por parte del profesor es esencial con el fin de mediar en las actividades cooperativas una participación equitativa, reconocimiento y evaluación a lo interno del grupo.

\section{Elementos esenciales en actividades cooperativas}

Los siguientes son los aspectos que se requieren para realizar actividades cooperativas en el aula.

1- Interdependencia positiva: los miembros de un grupo saben que los esfuerzos de cada integrante beneficiara a él mismo y también a los demás.

2- Responsabilidad individual: cada miembro deberá ser responsable de cumplir con la parte del trabajo que le corresponda.

3- La interacción y negociación: los alumnos deben realizar juntos el trabajo asignado, cooperando, negociando, apoyándose y motivándose unos a otros por su empeño en aprender.

4- Habilidades interpersonales y grupales: el aprendizaje cooperativo invita a los alumnos a que aprendan temas académicos así como habilidades interpersonales necesarias para funcionar en grupo. Así, los miembros del grupo deben saber cómo ejercer liderazgo, comunicarse, tomar decisiones y manejar los conflictos.

5- Evaluación grupal: los miembros del grupo deben analizar si se están alcanzando los objetivos y metas planteadas. Los grupos deben determinar qué acciones de sus miembros son positivas o negativas, y decidir qué conductas conservar o modificar (Johnson y Johnson, 1999, p.13).

Es compartido el aporte relevante del aprendizaje cooperativo al servicio de un ambiente de aula efectivo. La siguiente tabla resume algunas de sus características: 
Tabla 1. Características del aprendizaje cooperativo.

\begin{tabular}{|l|}
\hline Características del aprendizaje cooperativo \\
\hline Interés por el progreso de todos los actores del proceso de aprendizaje \\
\hline Responsabilidad individual \\
\hline Grupos heterogéneos \\
\hline Liderazgo compartido \\
\hline Aprendizaje al máximo posible \\
\hline Monitoreo e intervención constante del facilitador \\
\hline Abordaje de habilidades sociales \\
\hline
\end{tabular}

Fuente: Adaptado de García López, 1996 (citado por Traver J, 2003, p. 7).

\section{El aprendizaje cooperativo como perspectiva inclusiva}

El aprendizaje cooperativo ha sido objeto de numerosas investigaciones y estudios que constatan que este es efectivo para promover un aprendizaje significativo en interacción con los demás. Considerando este supuesto, es meritorio utilizar este tipo de aprendizaje en pro de la educación inclusiva ya que promueve la participación equitativa para que todos los miembros del equipo tengan las mismas oportunidades, potenciando al máximo el desarrollo de aspectos claves relacionados con su propio proceso de aprendizaje, favoreciendo así la autorregulación del aprendizaje, el compromiso, la negociación, la participación, la comunicación, la empatía, el apoyo mutuo y el respeto.

El objetivo primordial de este tipo de aprendizaje es la construcción de nuevo conocimiento con la contribución de otros, beneficiando a los estudiantes en diversas dimensiones (asiste a los estudiantes que tienen dificultades y enriquece a aquellos más aventajados). El trabajo cooperativo aborda, exitosamente, la diversidad del aula y constituye un acercamiento hacia prácticas académicas cada vez más inclusivas y menos segregativas o diferenciadoras.

Tal como lo describe Durán (2003), los métodos de aprendizaje cooperativo resultan idóneos en la promoción de la inclusión ya que estos se basan en la heterogeneidad de los alumnos, la diversidad es aprovechada en la instrucción académica (p. 1). 
Esta práctica académica abordada desde las aulas universitarias preparará a los y las estudiantes sin distinción de capacidades o habilidades para su inserción exitosa en la dinámica universitaria y posteriormente como profesionales en un contexto social diverso.

Pujolàs (2006) destaca ciertas ventajas al utilizar este tipo de aprendizaje. Estas ventajas constituyen oportunidades académicas para promover en las situaciones de aprendizaje la equidad y la inclusión:

- $\quad$ El alumnado se siente seguro al realizar tareas académicas.

- Se promueve la apreciación a la diversidad, se potencian las capacidades individuales como fuertes aspectos en la resolución de tareas.

- $\quad$ Existe un clima de participación y aceptación.

- La autoestima se eleva al saber que se está creando algo valioso y que se valoran positivamente cada contribución hecha al grupo.

- $\quad$ Cada alumno refuerza su identidad personal y cultural al sentirse un miembro valioso del grupo, no sólo por similitudes compartidas con los demás, sino también por aquellas características que le son únicas y personales.

- $\quad$ Facilita la comunicación intercultural, posibilitando la ruptura de estereotipos y prejuicios que favorezcan el acercamiento y la aceptación de todos los miembros del aula.

- $\quad$ El rendimiento académico es más elevado por parte de todos los alumnos, se practica el razonamiento y pensamiento crítico, hay mayor motivación para realizar las tareas asignadas.

- La cooperación da lugar a relaciones más positivas, relaciones solidarias y comprometidas entre los estudiantes.

- La cooperación produce mayor integración social, mejora la autoestima individual y refuerza la capacidad para enfrentar la adversidad y las tensiones. (pp. 34-35).

\section{Motivación hacia la cooperación en el aula universitaria}

El trabajo en equipo enfocado a la cooperación entre estudiantes con características diferentes requiere un esfuerzo motivador. En nuestra experiencia, hemos constatado situaciones negativas en las cuales los estudiantes no les agrada cooperar con otros estudiantes, pues mantienen criterios erróneos y excluyentes. En este sentido, el rol del profesor es 
vital, pues es este quien persuadirá y encantará a los estudiantes a beneficiarse del trabajo cooperativo ya que entre sus beneficios está el experimentar, a nivel individual, una serie de ventajas como lo es la construcción y reforzamiento de conocimientos, desarrollo de habilidades comunicativas, afectivas, creativas e imaginativas, entre otros aspectos valorativos.

El conocimiento se origina desde la interacción social y esta interacción es la que impulsa el desarrollo cognitivo, de modo que actividades de aprendizaje que se conciben desde el aprendizaje cooperativo como el explicar al compañero(a) representa una oportunidad para ejercitar lo aprendido, intercambiar opiniones, resolver problemas, clarificar dudas, compartir ideas o conocimientos, dialogar, comparar interpretaciones diversas, defender interpretaciones, cooperar y motivar a los demás compañeros(as) incrementan la comprensión y la adquisición en el individuo.

Pujolàs (2006) puntualiza ciertas condiciones necesarias en actividades cooperativas, éstas se consideran orientaciones generales para colaborar en el aula en pro de la inclusión, las cuales se mencionan a continuación.

- El aprendizaje en equipo debe ser puesto en práctica constantemente.

- $\quad$ El docente designa los grupos.

- El profesor comienza moderando la discusión y dando instrucciones.

- Cada miembro del grupo es responsable no sólo de colaborar con sus fortalezas sino también de ayudar a los otros a descubrir sus propias fortalezas. Cada estudiante fortalece al equipo con sus particularidades.

- Los estudiantes inseguros son alentados activamente a colaborar.

- $\quad$ El aprendizaje se ve influenciado en forma positiva con perspectivas diversas enriqueciendo la actividad académica.

- Los estudiantes son guiados a plantear objetivos claros, definiendo como se comunicarán, tomarán decisiones, resolverán conflictos y evaluarán su progreso.

- Los miembros del grupo tienen una doble responsabilidad: aprender lo planeado por el profesor y contribuir a que los demás también aprendan y sean partícipes activos. 
- $\quad$ Se diseñan actividades que promuevan el deseo de cooperar para aprender y el aprender a cooperar (p. 33).

Desde la generación de estas oportunidades y prácticas académicas los estudiantes aprenden a generar su propio conocimiento, enriqueciendo y fortaleciendo a cada alumno y alumna.

\section{Experiencia de inclusión: práctica cooperativa de tutorías estudiantiles}

Como profesoras de una lengua extranjera, en específico del idioma inglés, hacemos uso de múltiples actividades didácticas para lograr que los educandos logren adquirir el conocimiento y aprendan de manera significativa, entretenida y en cooperación con los demás; es decir, mediante trabajo en equipo. Una de las dificultades encontradas con mayor frecuencia al momento de aplicar una actividad que implique el trabajo en conjunto con otros compañeros o compañeras, es conformar los grupos de trabajo. Esto por cuanto los y las estudiantes muestran afinidad por trabajar con unos más que con otros, es ahí donde encontramos algún estudiante que se queda solo, sin equipo, y no es de extrañar que este educando sea el que ha presentado más dificultad en algún área de estudio específico. Esta fue la experiencia que tuvimos en el II ciclo lectivo del año 2013, cuando contamos entre los aprehendientes, a un estudiante de la carrera de Gestión Empresarial del Turismo Sostenible con dificultades de aprendizaje. Sus compañeros y compañeras mostraron, en un principio, renuencia a trabajar con este estudiante. Algunos dijeron que eso significaba trabajar "prácticamente solos". De modo que se realizaron ajustes e iniciativas que permitirán implementar actividades tendientes a fortalecer los valores como la solidaridad y la cooperación. Esta práctica académica requirió diseñar cada actividad de manera estructurada y con el claro objetivo de incluir a todos y todas en las actividades y sin contemplar agrupaciones por afinidad. Entonces, se motivó más el trabajo en grupos, guiando a todos hacia el aprendizaje.

De este modo, se procedió a investigar prácticas inclusivas que favorecieran la mediación de los aprendizajes asíque se plantearon grupos de trabajo distintos para cada tarea asignada. Por ejemplo, algunas de las actividades que implicó más interacción grupal fueron las visitas de campo para entrevistar en inglés a turistas internacionales. Igualmente, 
prácticas en empresas donde debían implementar actividades para solventar un problema. También surgió de manera espontánea y entre los mismos aprehendientes, el aprendizaje entre estudiantes o pares, el cual, a raíz de esta experiencia, hicimos la propuesta de su práctica de manera estructurada. El resultado final de esta experiencia en el II ciclo, 2013 fue muy satisfactoria ya que se logró consolidar y unir al grupo del curso. Los estudiantes comprendieron que a veces les toca ayudar a aprender y en otras les corresponde ser ayudados en su proceso de aprendizaje.

Esta experiencia ha motivado nuestro interés en promover e implementar prácticas académicas inclusivas, puntualizando la efectividad constatada en el grupo mencionado anteriormente, mediante el proceso de aplicación de actividades cooperativas y sobre todo de las tutorías entre pares o tutorías estudiantiles.

\section{Las tutorías entre pares: un aprendizaje efectivo e inclusivo}

La tutoría se define como la ayuda y el apoyo que brindan personas que no son maestros profesionales en el aprendizaje, de otros de una manera interactiva, decisiva y sistemática. Generalmente se efectúa de persona a persona, en pareja (Topping, 2000, p. 7).

Para Mosca y Santiviago (2009), las tutorías entre pares, surgen por la interacción. Esta interacción es la que hace posible el conocimiento, producto de la vivencia y en el encuentro con otros. Las tutorías entre estudiantes o pares fomentan un vínculo entre el estudiante - tutor y el aprendiz que permite compartir conocimiento, por un lado, el estudiante tutor obtiene un conocimiento profundo de un contenido específico y por el otro, el estudiante aprendiz adquiere, aclara y reafirma ese conocimiento (p.10).

Este intercambio no solo se circunscribe al aspecto cognitivo, sino también se refiere al aspecto social ya que promueve una relación entre iguales de manera amistosa, compartiendo su energía y entusiasmo. Existe un involucramiento por ambas partes, las cuales poseen un mismo código de comunicación, es decir, hablan un mismo idioma al compartir intereses propios de su generación. Esto facilita el aprendizaje y lo hace más personal y gratificante al darse de amigo a amigo.

El beneficio para ambos estudiantes a nivel emocional es considerable, por tanto, al ser un aprendizaje más independiente, 
genera una experiencia de exploración al cómo aprendo, lo que los hace autónomos, con su correspondiente contribución al crecimiento personal y a su propia madurez.

A favor de esta efectividad en el proceso de aprendizaje percibido desde esta perspectiva cooperativa Topping (1996), establece una tipología de las tutorías desde varias dimensiones, afirmando que la asignación de roles es fundamental en las tutorías entre estudiantes, donde hay un tutor y una persona por tutelar. Esta asignación de roles debe rotar de manera estructurada y con continuidad, de tal manera, que todos los y las estudiantes, tengan oportunidad de ser tutores, o bien, ser tutelados. Por ello, se recomienda que a pesar de que los estudiantes pueden pertenecer a diferentes años o nivel de educación, también es efectivo que los niveles de conocimiento no sean muy distantes entre el tutor y el estudiante tutelado (pp. 324 - 325). En este sentido, el autor señala que no necesariamente, el estudiante tutor debe ser más talentoso ya que las tutorías no solo buscan consolidar aprendizajes académicos, sino también promueven el desarrollo afectivo, emocional, actitudinal y social del educando a través de la cooperación. En la figura 2, se ilustra las dimensiones de las tutorías.

\section{Figura 2: Tipología de las Tutorías}

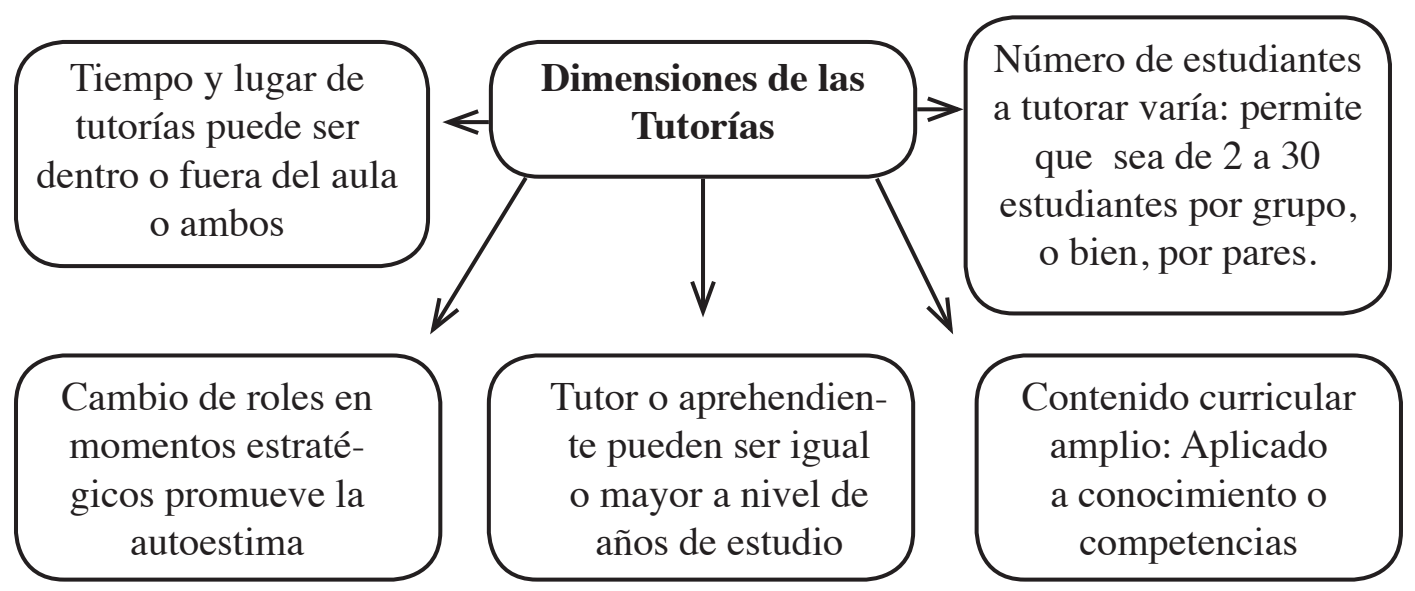

Fuente: Adaptación de Topping (1996). 
Las tutorías entre pares permiten que se practiquen métodos de enseñanza -aprendizaje ya experimentados por los mismos estudiantes, aplicando los que ya les han funcionado, lo que disminuye la posibilidad de que el educando se sienta intimidado y ofuscado ante el riesgo de frustración. Entonces, con este tipo de prácticas se puede asegurar una mayor participación de los estudiantes involucrados, promoviendo el hecho de que sea más proactivo en el aula.

Topping (2000) señala que la enseñanza mediante tutoría promueve un aprendizaje efectivo desde diferentes formas para distintas parejas (p. 6).

La tabla dos puntualiza los efectos de la práctica de las tutorías entre estudiantes o pares.

Tabla 2: Efectos de la práctica de tutorías entre pares.

\begin{tabular}{|c|c|c|}
\hline Acompañamiento & Oportunidades & Cooperación \\
\hline $\begin{array}{ll}\text { _ } & \text { Mayor ayuda individua- } \\
& \text { lizada } \\
\text { - } & \text { Mayor apoyo } \\
\text { - } & \text { Mayor motivación y au- } \\
\text { tocrítica } \\
\text { - } \\
\text { Más retroalimentación } \\
\text { inmediata y reconoci- } \\
\text { mientos }\end{array}$ & $\begin{array}{ll}\text { _ } & \text { Más actividad y variedad } \\
\text { - } & \text { Mayor ejercicio práctico } \\
\text { - } & \text { Más ejemplos particulares } \\
\text { y de mayor relevancia } \\
\text { - } \\
\text { Más autorregulación y } \\
\text { apropiamiento del proceso } \\
\text { de aprendizaje }\end{array}$ & $\begin{array}{ll}\text { _ } & \text { Más crítica o cues- } \\
\text { tionamiento } \\
\text { - } & \text { Un vocabulario } \\
\text { simplificado } \\
\text { - } \\
\text { Más dudas disipa- } \\
\text { das } \\
\text { - } \\
\text { Mejor autoconoci- } \\
\text { miento de la propia } \\
\text { manera de aprender. }\end{array}$ \\
\hline
\end{tabular}

Fuente: Adaptado de Topping (2000).

\section{Aplicación de las tutorías estudiantiles o entre pares}

Las tutorías entre estudiantes o pares o tutorías estudiantiles maximizan el aprendizaje a través de una interacción estructurada entre estudiante-estudiante, en vez de la tradicional instrucción continua que se deriva de la interacción entre el profesor y el estudiante. La actividad se describe en los siguientes pasos: 
1: $\quad$ El docente explica a los estudiantes de lo que se trata la actividad de tutorías entre pares o estudiantiles y sus beneficios. A su vez, establece parejas de trabajo de acuerdo con los perfiles observados durante el curso en cuestión para definir cuál estudiante será el tutor y cuál el aprendiz en cada par.

2: $\quad$ Se discuten objetivos y metas de aprendizaje incluyendo los surgidos por iniciativa de cada estudiante, por lo que se tienen tanto propósitos en común y también los que pudieran surgir como propios en cada pareja de estudiantes. Acá se planifican sesiones de trabajo, se programan actividades didácticas, clases y las evaluaciones formativas, todo de acuerdo con el área de conocimiento específica que se pretende estudiar.

3: El profesor explica los contenidos a repasar por cada pareja. Es necesario aclarar que los estudiantes tutores no introducirán temas, sino que revisarán y practicarán las áreas de conocimiento que han presentado mayor dificultad, aportando su propia metodología, materiales, herramientas, etc. necesarias para el aprendizaje.

4: $\quad$ Tanto el profesor como el estudiante tutor generan el material de trabajo o prácticas que les permita al estudiante aprendiz consolidar el conocimiento meta. Todo lo anterior, como parte del reforzamiento académico, producto de la experiencia académica de cada una de las partes.

5: Con la orientación previa del docente a cargo, el estudiante tutor diseña y programa las evaluaciones formativas que así se hayan establecido con anterioridad en el programa, contemplando también autoevaluaciones y coevaluaciones.

6: Tanto el estudiante tutor como el aprendiz, reportan al profesor y al grupo en general, el desempeño de cada una de las partes en el proceso, tanto durante como al finalizar el proceso, brindando recomendaciones específicas para lograr el éxito de la implementación de esta actividad didáctica. Igualmente, el docente, a través de la observación continua del desempeño de los estudiantes, obtendrá información valiosa que complementará con la que los propios estudiantes proporcionen en el reporte.

Es importante también mencionar que los roles asignados a los estudiantes son temporales ya que se pueden variar de acuerdo con los contenidos o áreas de estudio. Es decir, que acorde con las habilidades 
demostradas por los estudiantes para cada contenido, se pueden asignar nuevos roles, teniendo la oportunidad de que un estudiante sea el tutor de otro para ayudarle a que aprenda un área específica, a su vez, pueda ser que en otra oportunidad, sea él el aprendiz con otro conocimiento por consolidar. Asimismo, se le da oportunidad a las parejas de estudiantes a que en horario fuera de clase, se puedan reunir y trabajar en conjunto para así poder alcanzar las metas propuestas.

\section{El papel del docente}

El profesor a cargo es el facilitador y orientador del proceso y es el que acompaña a los estudiantes en todo momento, monitoreando el desempeño de ambos educandos en los roles asignados, dando asesoría, soporte y consultoría acerca de la planificación del proceso, los tiempos asignados a cada segmento y sobre las áreas del conocimiento a estudiar. Además, brinda una realimentación general sobre la experiencia al finalizar el proceso.

No está de más mencionar que el asignar estudiantes tutores en la clase, no significa tiempo libre para el docente a cargo. Todo lo contrario, el profesor es un elemento invaluable, integrador del proceso de aprendizaje cooperativo. Para ello, requiere de la habilidad y experiencia académica para saber agrupar cada pareja, estructurar toda la dinámica, caminar y asistir a cada pareja de estudiantes en su participación activa, dando sugerencias y asegurándose de que cada estudiante esté empleando las habilidades necesarias para llevar a cabo el proceso con éxito.

\section{Retos}

Las tutorías entre estudiantes o pares, lamentablemente, no es una práctica común en las aulas universitarias. Es una estrategia pedagógica especializada, por ello requiere invertir tiempo en su planificación y estructuración. Además, el profesor del curso a cargo requiere de conocer cómo son sus estudiantes; es decir, debe tomarse el tiempo para acercarse a ellos y conocer sus habilidades, fortalezas, por supuesto, sus debilidades. Este aspecto se dificulta cuando son grupos numerosos y muy heterogéneos en intereses y naturaleza en general.

Igualmente, el docente debe tomar un tiempo para enseñar a sus estudiantes las habilidades necesarias para enseñar, compartir su 
conocimiento y hacer conciencia de que ayudando a otros, también se aprende porque se profundiza el conocimiento. A su vez, es un tiempo para que el estudiante se conozca así mismo y así aprenda a maximizar su potencial.

Equivalentemente, la aplicación de las tutorías entre estudiantes o pares, exige también de una preparación y conocimiento cuidadoso por parte del profesor para agrupar idóneamente a los estudiantes en pares, definiendo quién deberá ser el tutor y quién, en ese momento específico, le corresponderá ser el aprendiz; todo de acuerdo con las necesidades y perfiles de cada educando.

\section{Otras actividades académicas tendientes a abordar la diversidad en el aula universitaria}

Los estudiantes y futuros profesionales ven en las aulas universitarias un ente que no siempre acoge y considera sus particularidades por lo que es imperativo lograr una constante, adaptar programas, recursos materiales y humanos para atender la diversidad que, de forma natural, posibilite y fomente la interacción entre todos los educandos y articulando, desde las clase, una metodología cooperativa - inclusiva que contemple actividades de aprendizaje tendientes a respetar las habilidades, gustos, capacidades, ritmos de aprendizaje, autonomía y estilos de aprendizaje de los y las estudiantes.

\section{TAI (grupo de asistencia individualizada)}

Esta técnica tiene como principal característica la combinación del aprendizaje cooperativo con la instrucción individualizada: todos los alumnos trabajan sobre lo mismo, pero cada uno de ellos siguiendo un programa específico. Es decir, la tarea de aprendizaje común se estructura en programas individualizados o, personalizados para cada miembro del equipo (Parrilla, 1992, citado por Pujolàs, 2002 p.13 ).

\section{Rompecabezas}

Esta es una actividad de cooperación en el tratamiento de variadas tareas académicas como resolución de problemas. En esta actividad, cada estudiante es esencial en la realización de tareas a completar. El 
Jigsaw fue utilizado por primera vez en 1971 en la Universidad de Texas por Elliot Aronson, como respuesta a los problemas raciales surgidos en el salón de clases. Aronson (2000, parraf. 1) describe la actividad en los siguientes pasos:

1. Dividir el aula en grupos de cinco o seis personas que se llamarán casa.

2. Asignar diferentes partes de una tarea de aprendizaje (5 a 6 componentes) a cada uno de los miembros del grupo. Las tareas pueden requerir leer, analizar literatura, resolver problemas o estudio de casos. Estas tareas deben requerir interacción y cooperación continua.

3. Cada estudiante se familiariza con el material y elabora un informe, a la vez, que se responsabiliza del aprendizaje de su componente asignado por parte del resto de miembros.

4. Luego se forman nuevos grupos de "expertos" (estudiantes de diferentes grupos asignados de una misma tarea) que deberán discutir, perfilar respuestas o propuestas y mejorar los informes individuales.

5. Después de esa discusión, se regresa al grupo "casa" donde cada experto explica y comparte su tarea asignada, o bien, se plantea la solución de la actividad o problema. Se interactúa aportando comentarios o preguntas. Cada parte expuesta por cada estudiante debe ser esencial para completar la tarea general y la total comprensión de la lección.

\section{Rotar a estaciones}

Esta actividad permite abordar cooperativamente un tema de estudio. En primerl lugar, se designan cuatro estaciones cada una con material y guía de trabajo. Se procede a explicar la clase, en la que participarán en un trabajo colaborativo completando una tarea específica (elaborada por el profesor) en la cual rotarán a diferentes estaciones previamente establecidas. La clase es dividida en cuatro grupos. Cada grupo se designa a una estación por un tiempo limitado.

En la estación, el grupo debe completar la actividad encontrada, procurando interactuar y dialogar sobre la misma con todos los miembros. Los estudiantes reportan y dejan comentarios o preguntas en la 
estación en relación con lo discutido. El profesor indica a los estudiantes cuando se deberán mover de estación y seguir las instrucciones en la guía de trabajo de la nueva estación. De igual forma, deben reaccionar a los comentarios y preguntas generadas por el grupo anterior. Así se continúa sucesivamente hasta que los grupos visiten todas las estaciones. Como conclusión se realiza una plenaria sobre los detalles que los estudiantes consideren relevantes. El profesor guía la discusión sobre detalles claves (Adaptado de Simon, 2014, Strategy in practice, parraf. 1).

\section{Conclusión}

En los tiempos en que vivimos, donde todo se presenta de manera vertiginosa y muy competitiva, se hace necesario hacer un alto en el camino para ejecutar prácticas que nos lleven a acercarnos y ayudarnos unos a otros. Los seres humanos somos diversos, diferentes, únicos y esta naturaleza, que muchos podrán decir complicada, nos hace ricos. Por ello, desde el aula universitaria, los docentes, tenemos la responsabilidad de maximizar estas distinciones en los estudiantes para sacar provecho de su potencial, apelando a nuestro sentido de solidaridad y cooperación y haciendo de esto, el punto de partida para cualquier actividad que emprendamos. Esta debe ser la referencia más fuerte.

El aprendizaje cooperativo y la educación inclusiva han ocupado poco espacio en la formación pedagógica de los educadores. Por lo tanto, su aplicación no ha tenido la relevancia que se requiere. Los distintos autores señalados en este artículo coinciden en la necesidad de implementar éstas prácticas en todo ámbito educativo que pretenda tener un carácter positivo, obteniendo transformaciones importantes en nuestra sociedad.

Entonces, la percepción de la educación como un acto meramente individual, propio de cada quien, ha venido cambiando para darle paso a un rol educativo con carácter social, correspondiente a la propia naturaleza humana. Y es que el ser humano aprende bajo el precepto del intercambio que se da entre todos los actores del proceso enseñanza-aprendizaje.

Por todo esto, la aplicación de prácticas que conlleven a conjuntarnos en un solo camino hacia el conocimiento y de la mano del aprendizaje colaborativo basado en prácticas didácticas inclusivas como el trabajo en equipo y las tutorías entre estudiantes o pares, es 
un ejercicio de intercambio en el que, como es la vida, hoy yo ayudo y luego, o simultáneamente, yo recibo ayuda. Es en esta "transacción" de experiencias y conocimientos que podemos mejorar como seres humanos, al mismo tiempo que nos formamos en el saber.

Sin embargo, aún falta por hacer. Se debe pasar de esta conciencia a la acción. Para ello se necesita información, mayor orientación y también muchas ganas de implementar actividades didácticas diversas, innovadoras que promuevan el aprendizaje cooperativo e inclusivo. Solo así lograremos un aprendizaje equitativo y de calidad que merecen nuestros estudiantes. Este es nuestro gran reto como educadores.

\section{Referencias bibliográficas}

Aronson, E. ( 2000). The jigsaw classroom. Recuperado de http://www. jigsaw.org/

Durán, D. 2003). Una experiencia de tutoría entre iguales, método de aprendizaje cooperativo para la diversidad. Trabajo presentado en el Congreso Internacional: una escuela para todos. Barcelona. Recuperado de http://www.aieteikastetxea.org/wp- content/ uploads/2011/07/ponenciablanquernacast1.pdf

Echeita, G. (2005). Perspectivas y dimensiones críticas en las políticas de atención a la diversidad. Revista Alambique: Didáctica de las ciencias experimentales, 44, 7-16. Recuperado de http:// www.uam.es/personal_pdi/stmaria/sarrio/DOCUMENTOS, $\% 20$ ARTICULOS, \%20PONENECIAS,/Atencion $\% 20 \mathrm{a} \% 201 \mathrm{a} \% 20$ diversidad.pdf

Johnson, D y Johnson, R. (1999). Aprender juntos y solos. Recuperado de http://terras.edu.ar/jornadas/3/biblio/3JOHNSON-David-JOHNSON-Roger-Apendice.pdf.

Kagan, S. (2001) Cooperative learning structures can increase student achievement. Recuperado de http://www.kaganonline.com/ free_articles/research_and_rationale/311/Cooperative-Learning-Structures-Can-Increase-Student-Achievement

Ley 7600. (1996). Igualdad de oportunidades para las personas con discapacidad. Recuperado de http://wvw.nacion.com/ln_ee/ESPECIALES/documentos/2003/mayo/29/ley.pdf

Meléndez. L. et al. (2012). IV Informe Estado de la Educación. Programa Estado de la Nación en Desarrollo Humano Sostenible. 
Informe Final. Desarrollo y Desafíos de las Adecuaciones Curriculares en el sistema educativo costarricense. Costa Rica. 4-120 Recuperado de http://www.estadonacion.or.cr/files/biblioteca virtual/educacion/004/melendez-et-al-adecuaciones-curriculares.pdf

Mosca, A y Santiviago, C. (2009). Tutorías entre pares. Recuperado de http://www2.compromisoeducativo.edu.uy/sitio/wp-content/ uploads/2013/10/librotutorias.pdf

Naradowski, M. (2008). La inclusión educativa. Reflexiones y propuestas entre las teorías, las demandas y los slogans. Revista Iberoamericana sobre Calidad, Eficacia y Cambio en Educación, 6(2),19-26. Recuperado de http://www.rinace.net/ arts/vol6num2/art2_htm.htm

Pujolàs, P. (2002) El aprendizaje cooperativo algunas propuestas para organizar de forma cooperativa el aprendizaje en el aula. Laboratorio de psicopedagogía. 3-39. Recuperado de http://www.deciencias.net/convivir/1.documentacion/D.cooperativo/AC_Propuetasorganizativas_Pujolas_39p.pdf

Pujolàs, P. (2006). Aulas inclusivas y aprendizaje cooperativo. Recuperado de http://www.octaedro.com/pdf/10062.pdf

Simon, C. (2014). Rotating reviews. Recuperado de http://www.readwritethink.org/professional-development/strategy-guides/brainstorming-reviewing-using-carousel-30630.html

Stainback, S. (2001). Classroom contextual considerations. Recuperado de http://www.sig2.hawaii.edu/resources/briefings/topic6/ articles.php

Topping. K. (1996). The effectiveness of peer tutoring in further and higher education: A typology and review of the literature. Recuperado de http://www.fau.edu/CLASS/CRLA/Level_Three/ The_effectiveness_of_peer_tutoring_in_further_and_higher_ education-a_typology_and_review_of_the_literature.pdf

Topping, K. (2000). Enseñanza: 5 prácticas educativas. Recuperado de http://www.ibe.unesco.org/publications/educationalpracticesseriespdf/prac $05 \mathrm{~s} . \mathrm{pdf}$

Traver, J. (2003). Aprendizaje cooperativo y educación intercultural. Revista Educación intercultural: La diversitat cultural a l'escola, 1-29. Recuperado de http://centros.edu.xunta.es/cfr/pontevedra/oblogdeorientacion/toni/toni5.pdf 
UNESCO. (2009). Directrices sobre políticas de inclusión en la educación. Recuperado de http://unesdoc.unesco.org/images/0017/001778/177849s.pdf 